\title{
Magnetoacoustic Heating in Nonisentropic Plasma Caused by Different Kinds of Heating-Cooling Function
}

\author{
Anna Perelomova \\ Gdansk University of Technology, Faculty of Applied Physics and Mathematics, Ul. Narutowicza 11/12, 80-233 Gdansk, Poland \\ Correspondence should be addressed to Anna Perelomova; anna.perelomova@pg.edu.pl
}

Received 25 July 2018; Accepted 2 September 2018; Published 24 September 2018

Academic Editor: Alexander Iomin

Copyright (c) 2018 Anna Perelomova. This is an open access article distributed under the Creative Commons Attribution License, which permits unrestricted use, distribution, and reproduction in any medium, provided the original work is properly cited.

\begin{abstract}
The nonlinear phenomena which associate with magnetoacoustic waves in a plasma are analytically studied. A plasma is an open system with external inflow of energy and radiation losses. A plasma's flow may be isentropically stable or unstable. The nonlinear phenomena occur differently in dependence on stability or instability of a plasma's flow. The nonlinear instantaneous equation which describes dynamics of nonwave entropy mode in the field of intense magnetoacoustic perturbations is the result of special projecting of the conservation equations in the differential form. It is analyzed in some physically meaningful cases; those are periodic magnetoacoustic perturbations and particular cases of heating-cooling function. A plasma is situated in the straight magnetic field with constant equilibrium magnetic strength which form constant angle with the direction of wave propagation. A plasma is initially uniform and equilibrium. The conclusions concern nonlinear effects of fast and slow magnetoacoustic perturbations and may be useful in direct and inverse problems.
\end{abstract}

\section{Introduction}

The study of propagation of magnetohydrodynamic (MHD) perturbations has a key role in the astrophysical applications. It is of crucial importance in solar hydrodynamics, in particular in formation of coronal loops, fluid flows in thermonuclear reactors, interstellar gases, and planetary nebulae. Gaseous plasma is an open system with disturbed adiabaticity of wave perturbations. This may be modeled by some heating-cooling function which reflects the physical conditions of energy inflow and radiation losses [1-3]. Linear dynamics of the MHD waves, that is, waves of infinitely small magnitude in an open plasma which may be isentropically unstable, has been well studied in the last decade. The main conclusions are common in hydrodynamics of open systems $[4,5]$. Flows of other fluids in thermodynamical nonequilibrium reveal analogous features which are described by the similar equations $[6,7]$. Apart from complexity of the MHD system of equations which imposes coexistence of slow, fast sound modes, and Alfvén modes, along with the nonwave modes, other linear effects go into play, such as mechanical damping and thermal conduction of a plasma.
The general nonlinear dynamics of perturbations in plasma is not far well understood. As for the peculiarities of nonlinear evolution of wave MHD perturbations without accounting for their interaction with other modes, they are well understood starting from some particular but important cases which concern one-dimensional flows along and across the magnetic field [8-10] and ending by various numerical investigations. Nakariakov and coauthors in [11] advanced in the analysis of one-dimensional propagation of the MHD wave perturbations at arbitrary angle between the straight magnetic field and direction of wave's propagation. They have derived the generic weakly nonlinear evolutionary equation which describes slow and fast perturbations in active plasma. Authors of [12] concluded about possibility of self-organization of MHD waves and described theoretically slow magnetoacoustic shock autowaves with magnitude independent on the initial or boundary conditions. They discovered that the extension of a heating-cooling function in the Taylor series accounting for quadratic nonlinear terms "introduces new physics such as existence of solitary waves". The conclusions concern nonlinear dynamics of individual slow and fast wave perturbations. The formation of the 
asymmetric autowave pulse in the similar geometry of a flow was described in [13].

While perturbations of infinitely small magnitude develop independently, nonlinearity makes different types of a fluid's motion to interact in physical reality which deals with finite-magnitude perturbations. The wave modes may excite nonwave modes which form inhomogeneities in turn impacting on wave propagation; waves may be scattered by other waves, and so on [14-16]. Apart from nonlinearity, the reasons for interaction are irreversible losses in energy and momentum or/and the disturbance of adiabaticity. In description of nonlinear interaction of modes, we face with mathematical difficulties much serious as compared to the case of nonlinear dynamics of individual mode. The nonlinear excitation of the entropy mode in the field of slow and fast magnetosonic perturbations for different kinds of external heating-cooling function is the subject of this study. Following Nakariakov and coauthors, we consider weakly nonlinear flow of completely ionized gas affected by some generic heating-cooling function. Weak nonlinearity means the smallness of the Mach number in a flow: $M \ll 1$. Also, the impact of nonlinearity may be obtained as corrections to the linear results. This concerns the links between specific perturbations inside every mode and dynamic equations. The method which has been applied by the author in studies of nonlinear interaction of wave and nonwave modes in a number of different fluid flows is useful in investigation of magnetic flows as well. It allows subdividing nonlinear dynamic equations for perturbations in a specific mode from the system of conservation equations in a differential form. The procedure is in fact some linear combination of equations which eliminates all liner terms containing nonspecific perturbations. The dynamic coupling equations include the first-order derivatives with respect to time. The quadratic nonlinear terms which are products of nonspecific perturbations and their derivatives form the "foreign forces". In the context of weak nonlinearity, the quadratic terms including magnetosonic perturbations are of importance. Nonlinear corrections in the dynamic equations for fast and slow magnetosonic perturbations may be also considered as a result of self-interaction of corresponding wave mode. Nonlinearity, even weak, accumulates in time and is responsible for formation of shock fronts, enrichment of perturbations' spectrum, and nonlinear transfer of energy and momentum. In regard to magnetoacoustic heating, nonlinear transfer of the wave energy into the energy of the entropy mode leads to forming of the thermal lenses which in turn scatter sound. The effects of plasma's boundaries are not considered. Nonlinear dynamic equations are valid with the accepted accuracy up to quadratic nonlinear terms, that is, up to terms proportional to the squared Mach number, $M^{2}$. That concerns also terms originating from the heating-cooling function. The perturbations which specify the entropy mode enhance with time. In the frames of a model, their magnitudes should not exceed magnitudes of corresponding wave perturbations. When these magnitudes equalize, the entropy mode may have impact on the wave process. Hence, the equations are valid over some temporal and spatial domains, where wave perturbations remain dominant.
One may expect that not only the wave processes, but the magnetoacoustic heating reveals anomalous behavior in the isentropically unstable active plasma, as it happens to all acoustically active media independently on the physical reason of acoustical activity [6,7]. The subsequent analysis confirms this conclusion. We do not consider mechanical and thermal losses in a plasma and its finite electrical conductivity which are well studied. They introduce additional attenuation and dispersion. The magnetoacoustic heating due to propagation of the MHD perturbations across the straight magnetic field has been studied by the author in $[17,18]$. In this study, we analyze the instantaneous dynamic equation of excitation of the entropy mode by fast and slow MHD sound modes in the field of planar waves which form an arbitrary angle with the magnetic strength and discuss it in some physically meaningful cases of wave perturbations and the heating-cooling function. That concerns also the case of nonadiabatic instability. The first results in a special case of heating-cooling function which depends exclusively on temperature may be found in the recent author's paper [19]. In this study, we make conclusions about entropy perturbations due to magnetosonic heating in general case.

\section{Perturbations of Infinitely Small Magnitude in the MHD Flow}

The system of PDE equations consists of continuity equation, momentum equation for ionized gas, energy balance equation, and electrodynamic equations:

$$
\begin{aligned}
\frac{\partial \rho}{\partial t}+\vec{\nabla} \cdot(\rho \vec{v}) & =0, \\
\rho \frac{d \vec{v}}{d t} & =-\vec{\nabla} p+\mu_{0}(\vec{\nabla} \times \vec{B}) \times \vec{B}, \\
\frac{d p}{d t}-\gamma \frac{p}{\rho} \frac{d \rho}{d t} & =(\gamma-1) L(p, \rho), \\
\frac{\partial \vec{B}}{\partial t} & =\vec{\nabla} \times(\vec{v} \times \vec{B}), \\
\vec{\nabla} \cdot \vec{B} & =0,
\end{aligned}
$$

where $\vec{v}, \vec{B}, p$, and $\rho$ are the velocity of a plasma, the magnetic field strength, plasma's pressure, and density, respectively, and $\mu_{0}$ is the magnetic permeability of free space. $L$ is the heating-cooling function which disturbs adiabaticity of wave perturbations in a plasma. The choice of the heating-cooling function which depends on pressure and density makes using these basic thermodynamic variables and expressing all other thermodynamic quantities such as internal energy as functions of pressure and density convenient. Following [11], it is assumed to be dependent on pressure and density. The third equation in the set (1) relates to an ideal gas with the caloric equation of state

$$
e=\frac{p}{(\gamma-1) \rho},
$$




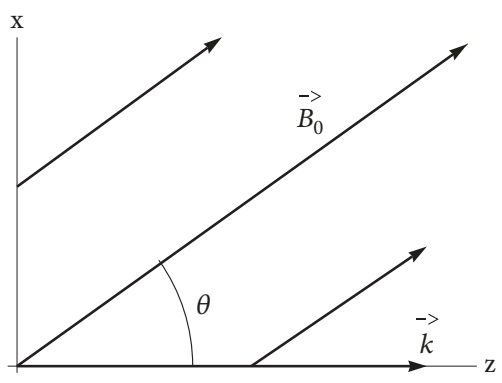

FIgURE 1

where $e$ denotes the internal energy of a gas and $\gamma=C_{P} / C_{V}$ is the ratio of specific heats under constant pressure and constant density. We consider the same geometry of a flow as in [11]. The equilibrium magnetic strength $\vec{B}_{0}$ lies in the $(x, z)$ plane. Its equilibrium magnitude $B_{0}$ is constant, and it forms constant angle $\theta$ with the positive direction of axis $z$, so

$$
\begin{aligned}
& B_{0, x}=B_{0} \sin (\theta), \\
& B_{0, z}=B_{0} \cos (\theta)
\end{aligned}
$$

denote projections of $\vec{B}_{0}$ onto axis $x$ and $z$. Axis $z$ points to the direction of wave propagation. Figure 1 recalls the geometry of a planar flow.

All field quantities are expanded in the vicinity of the equilibrium state as $f(z, t)=f_{0}+f^{\prime}(z, t)$. We consider initially homogeneous stationary plasma without bulk flows. That imposes $v_{0, x}=v_{0, y}=v_{0, z}=0$. The starting point represents the leading-order equations which follow from (1):

$$
\begin{aligned}
& \frac{\partial \rho^{\prime}}{\partial t}+\rho_{0} \frac{\partial v_{z}}{\partial z}=-\rho^{\prime} \frac{\partial v_{z}}{\partial z}-v \frac{\partial \rho^{\prime}}{\partial z} \\
& \frac{\partial v_{x}}{\partial t}-\frac{B_{0, z}}{\rho_{0} \mu_{0}} \frac{\partial B_{x}^{\prime}}{\partial z}=-\frac{B_{0, z}}{\rho_{0} \mu_{0}} \rho^{\prime} \frac{\partial B_{x}^{\prime}}{\partial z}-v_{z} \frac{\partial v_{x}}{\partial z}, \\
& \frac{\partial v_{y}}{\partial t}-\frac{B_{0, z}}{\rho_{0} \mu_{0}} \frac{\partial B_{y}^{\prime}}{\partial z}=-\frac{B_{0, z}}{\rho_{0} \mu_{0}} \rho^{\prime} \frac{\partial B_{y}^{\prime}}{\partial z}-v_{z} \frac{\partial v_{y}}{\partial z}, \\
& \frac{\partial v_{z}}{\partial t}+\frac{1}{\rho_{0}} \frac{\partial p^{\prime}}{\partial z}+\frac{B_{0, x}}{\rho_{0} \mu_{0}} \frac{\partial B_{x}^{\prime}}{\partial z} \\
& =-\frac{B_{0, z}}{\rho_{0} \mu_{0}} \rho^{\prime} \frac{\partial B_{x}^{\prime}}{\partial z}-\frac{\rho^{\prime}}{\rho_{0}} \frac{\partial p^{\prime}}{\partial z}-v_{z} \frac{\partial v_{z}}{\partial z} \\
& -\frac{1}{2 \rho_{0} \mu_{0}} \frac{\partial}{\partial z}\left(B_{x}^{\prime 2}+B_{y}^{\prime 2}\right) \text {, } \\
& \frac{\partial p^{\prime}}{\partial t}+c_{0}^{2} \rho_{0} \frac{\partial v_{z}}{\partial z}-(\gamma-1)\left(L_{p} p^{\prime}+L_{\rho} \rho^{\prime}\right) \\
& =-v_{z} \frac{\partial p^{\prime}}{\partial z}-\gamma p^{\prime} \frac{\partial v_{z}}{\partial z} \\
& +(\gamma-1)\left(0.5 L_{p p} p^{\prime 2}+0.5 L_{\rho \rho} \rho^{\prime 2}+L_{p \rho} p^{\prime} \rho^{\prime}\right)
\end{aligned}
$$

$$
\begin{aligned}
& \frac{\partial B_{x}^{\prime}}{\partial t}+\frac{\partial}{\partial z}\left(B_{0, x} v_{z}-B_{0, z} v_{x}\right)=-v_{z} \frac{\partial B_{x}^{\prime}}{\partial z}-B_{x}^{\prime} \frac{\partial v_{z}}{\partial z} \\
& \frac{\partial B_{y}^{\prime}}{\partial t}-B_{0, z} \frac{\partial v_{y}}{\partial z}=-v_{z} \frac{\partial B_{y}^{\prime}}{\partial z}-B_{y}^{\prime} \frac{\partial v_{z}}{\partial z}
\end{aligned}
$$

where

$$
\begin{gathered}
L_{p}=\frac{\partial L}{\partial p}, \\
L_{\rho}=\frac{\partial L}{\partial \rho}, \\
L_{p p}=\frac{\partial^{2} L}{\partial p^{2}}, \\
L_{\rho \rho}=\frac{\partial^{2} L}{\partial \rho^{2}}, \\
L_{p \rho}=\frac{\partial^{2} L}{\partial p \partial \rho}
\end{gathered}
$$

are partial derivatives of the heating-cooling function $L(p, \rho)$ evaluated at equilibrium state $\left(p_{0}, \rho_{0}\right)$ and

$$
c_{0}=\sqrt{\frac{\gamma p_{0}}{\rho_{0}}}
$$

is the acoustic speed in unmagnetized gas in equilibrium.

As usual, establishment of the dispersion relations is the primary procedure in the linear flows of a fluid. All perturbations are thought as a sum of planar waves proportional to $\exp \left(i \omega\left(k_{z}\right) t-i k_{z} z\right)$, where $k_{z}$ designates the wave number of any individual planar wave which propagates along axis $z$ :

$$
f^{\prime}(z, t)=\int_{-\infty}^{\infty} \tilde{f}\left(k_{z}\right) \exp \left(i \omega\left(k_{z}\right) t-i k_{z} z\right) d k_{z}
$$

and $\left(\tilde{f}\left(k_{z}\right) \exp \left(i \omega\left(k_{z}\right) t\right)=\tilde{f}\left(k_{z}, t\right)\right.$ denotes the Fourier transform of $f^{\prime}(z, t)$, so $\left.\tilde{f}\left(k_{z}, t\right)=(1 / 2 \pi) \int_{-\infty}^{\infty} f(z, t) e^{i k_{z} z} d z\right)$. The dispersion relations in a planar flow of a magnetic fluid reflect the solvability of (4). They take the form of seven solutions to the algebraic equation which demands zero main determinant of the system of linear equations:

$$
\begin{aligned}
\omega_{1,2} & = \pm \frac{B_{0, z} k_{z}}{\sqrt{\mu_{0} \rho_{0}}}= \pm C_{A, z} k_{z}, \\
\omega_{3,4,5,6} & =C k_{z}+i \frac{S}{c_{0}^{2}}\left(c_{0}^{2} L_{p}+L_{\rho}\right), \\
\omega_{7} & =\frac{i(\gamma-1) L_{\rho}}{c_{0}^{2}},
\end{aligned}
$$


where

$$
\begin{aligned}
S & =-\frac{\left(C^{2}-C_{A}^{2}\right)(\gamma-1)}{2\left(2 C^{2}-c_{0}^{2}-C_{A}^{2}\right)}, \\
C_{A} & =\frac{B_{0}}{\sqrt{\mu_{0} \rho_{0}}}, \\
C_{A, z} & =C_{A} \cos (\theta) .
\end{aligned}
$$

$C_{A}$ designates the Alfvén speed, $C_{A, z}$ is its $z$-component, and $C$ is one from four roots of the equation

$$
B_{0, x}^{2} C^{4}+\left(C^{2}-c_{0}^{2}\right)\left(B_{0, z}^{2}-\mu_{0} \rho_{0} C^{2}\right)=0 .
$$

All magnetosonic speeds are evaluated at the equilibrium state $\left(p_{0}, \rho_{0}\right)$. Four roots $C$ are real values for any $\theta$. With accounting for equalities

$$
\begin{aligned}
& \frac{B_{0, x}}{B_{0}}=\frac{C_{A, x}}{C_{A}}=\sin (\theta), \\
& \frac{B_{0, z}}{B_{0}}=\frac{C_{A, z}}{C_{A}}=\cos (\theta),
\end{aligned}
$$

(10) which determines acoustic speeds takes the form

$$
C^{4}-C^{2}\left(c_{0}^{2}+C_{A}^{2}\right)+c_{0}^{2} C_{A, z}^{2}=0 .
$$

The first two roots $\omega_{1}, \omega_{2}$ relate to the magnetic Alfvén waves, the next four roots specify magnetosonic waves of different directions of propagation (the so-called slow and fast MHD waves of different direction of propagation), and the last root $\omega_{7}$ corresponds to the entropy mode. The dispersion relations in (8) are calculated with accuracy up to terms proportional to the first powers of $L_{p}, L_{\rho}$. They have been established by Nakariakov and coauthors [11, 12] and were in fact the starting point for analysis of individual weakly nonlinear wave perturbations in a plasma. The condition of acoustic (isentropic) instability is

$$
c_{0}^{2} L_{p}+L_{\rho}>0,
$$

since $S$ is real negative value for any $C$ and $\theta$, apart form the particular case of $\theta=0$, when $C^{2}$ equals $C_{A}^{2}$ and $S=0$. This condition has been discovered in the earlier studies of nonequilibrium processes in fluids $[4,5]$. In the case of isentropic instability, the MHD wave perturbations of infinitely small magnitude enhance in the course of propagation. The condition of thermal isobaric instability sounds as

$$
L_{\rho}<0 .
$$

Note that the heating-cooling function is supposed to depend on pressure and density. The choice of the reference thermodynamic variables evidently impacts on the form of instability conditions. These inequalities are common for open systems and do not certainly overlap.

The linearized system (4) has an equivalent form

$$
\frac{\partial \widetilde{\psi}}{\partial t}+\widetilde{L} \widetilde{\psi}=(i \omega+\widetilde{L}) \widetilde{\psi}=0,
$$

where

$$
\begin{aligned}
& \widetilde{\psi}=\left(\begin{array}{c}
\widetilde{\rho} \\
\widetilde{v}_{x} \\
\widetilde{v}_{y} \\
\widetilde{v}_{z} \\
\widetilde{p} \\
\widetilde{B}_{x} \\
\widetilde{B}_{y}
\end{array}\right), \\
& \widetilde{L}=\left(\begin{array}{ccccccc}
0 & 0 & 0 & -i \rho_{0} k_{z} & 0 & 0 & 0 \\
0 & 0 & 0 & 0 & 0 & \frac{i k_{z} B_{0, z}}{\rho_{0} \mu_{0}} & 0 \\
0 & 0 & 0 & 0 & 0 & 0 & \frac{i k_{z} B_{0, z}}{\rho_{0} \mu_{0}} \\
0 & 0 & 0 & 0 & -\frac{i k_{z}}{\rho_{0}} & -\frac{i k_{z} B_{0, x}}{\rho_{0} \mu_{0}} & 0 \\
-(\gamma-1) L_{\rho} & 0 & 0 & -i k_{z} c_{0}^{2} \rho_{0} & -(\gamma-1) L_{p} & 0 & 0 \\
0 & i k_{z} B_{0, z} & 0 & -i k_{z} B_{0, x} & 0 & 0 & 0 \\
0 & 0 & i k_{z} B_{0, z} & 0 & 0 & 0 & 0
\end{array}\right)
\end{aligned}
$$


denote vector of the Fourier transforms of perturbations and matrix operating in the space of Fourier transforms. In fact, seven roots of dispersion equation (8) are the eigenvalues of matrix $i \widetilde{L}$. The total perturbation is represented by a sum of specific disturbances which are eigenvectors of $i \widetilde{L}$ corresponding to seven eigenvalues. Magnetoacoustic modes are represented by four eigenvectors which connect the Fourier transforms of specific perturbations:

$$
\widetilde{\psi}_{3,4,5,6}=\left(\begin{array}{c}
\tilde{\rho} \\
\tilde{v}_{x} \\
\widetilde{v}_{y} \\
\widetilde{v}_{z} \\
\widetilde{p} \\
\widetilde{B}_{x}^{\prime} \\
\widetilde{B}_{y}^{\prime}
\end{array}\right)_{3,4,5,6}=\left(\begin{array}{c}
1 \\
-\frac{B_{0, z}\left(C c_{0}^{2}\left(C^{2}-c_{0}^{2}\right) k_{z}+\left(i c_{0}^{2}(\gamma-1)+i S\left(C^{2}+c_{0}^{2}\right)\right)\left(c_{0}^{2} L_{p}+L_{\rho}\right)\right)}{B_{0, x} C^{2} c_{0}^{2} k_{z} \rho_{0}} \\
0 \\
\frac{C c_{0}^{2} k_{z}+i S\left(c_{0}^{2} L_{p}+L_{\rho}\right)}{k_{z} c_{0}^{2} \rho_{0}} \\
\frac{C c_{0}^{2} k_{z}-i(\gamma-1)\left(c_{0}^{2} L_{p}+L_{\rho}\right)}{C k_{z}} \\
\left.C c_{0, x}^{2}\left(C^{2}-c_{0}^{2}\right) k_{z}+i\left(c_{0}^{2}(\gamma-1)+2 S C^{2}\right)\left(c_{0}^{2} L_{p}+L_{\rho}\right)\right) \mu_{0} \\
0
\end{array}\right) \tilde{\rho}_{3,4,5,6}
$$

where the ordering number by $C$ is omitted. The relations between perturbations may be readily written on in $z, t$ variables, reminding that the factor $-i k_{z}$ corresponds to the operator $\partial / \partial z$ and $\left(-i k_{z}\right)^{-1}$ corresponds to $\int d z$. The upper limit of integration is $z$, and the lower one depends on the physical context of a flow. In the case $B_{0, x}=0$, the magnetoacoustic $\mathrm{x}$-component of velocity and perturbation in magnetic strength in all four modes are zero: $\widetilde{v}_{x}=0, \widetilde{B}_{x}=$ 0 , so $v_{x}=0, B_{x}^{\prime}=0$. The specific perturbations representing the Alfvén modes are linked in the following way:$$
\psi_{1}=\left(\begin{array}{c}
\rho^{\prime} \\
v_{x} \\
v_{y} \\
v_{z} \\
p^{\prime} \\
B_{x}^{\prime} \\
B_{y}^{\prime}
\end{array}\right)_{1}=\left(\begin{array}{c}
0 \\
0 \\
1 \\
0 \\
0 \\
-\frac{B_{0}}{C_{A}} \\
0
\end{array}\right) v_{y, 1},
$$$$
\psi_{2}=\left(\begin{array}{c}
0 \\
0 \\
1 \\
0 \\
0 \\
\frac{B_{0}}{C_{A}} \\
0
\end{array}\right) v_{y, 2}
$$

The entropy mode is established by the relations

$$
\psi_{7}=\left(\begin{array}{c}
\rho^{\prime} \\
v_{x} \\
v_{y} \\
v_{z} \\
p^{\prime} \\
B_{x}^{\prime} \\
B_{y}^{\prime}
\end{array}\right)_{7}=\left(\begin{array}{c}
1 \\
\frac{(\gamma-1) C_{A, x} L_{\rho}}{C_{A, z} c_{0}^{2} \rho_{0}} \int d z \\
0 \\
\frac{(\gamma-1) L_{\rho}}{c_{0}^{2} \rho_{0}} \int d z \\
0 \\
0 \\
0
\end{array}\right) \rho_{7} .
$$

All eigenvectors are derived with accuracy up to terms including the first powers of $L_{p}$ and $L_{\rho}$. The case $C_{A, z}=0$ corresponds to zero $v_{x}$ in the entropy mode. Any total perturbation is a sum of specific disturbances:

$$
\begin{aligned}
& v_{x}=\sum_{i=1}^{7} v_{x, i}, \\
& v_{y}=\sum_{i=1}^{7} v_{y, i}, \\
& v_{z}=\sum_{i=1}^{7} v_{z, i}, \\
& B_{x}^{\prime}=\sum_{i=1}^{7} B_{x, i}^{\prime}, \\
& B_{y}^{\prime}=\sum_{i=1}^{7} B_{y, i}^{\prime},
\end{aligned}
$$




$$
\begin{aligned}
& p^{\prime}=\sum_{i=1}^{7} p_{i}, \\
& \rho^{\prime}=\sum_{i=1}^{7} \rho_{i} .
\end{aligned}
$$

Index in summation $i$ denotes the ordering number of specific mode. The row which distinguishes individual excess

$$
P_{7}=\left(1-\frac{(\gamma-1) C_{A, x} \rho_{0}}{C_{A, z} c_{0}^{4}}\left(c_{0}^{2} L_{p}+L_{\rho}\right) \int d z \quad 0-\frac{(\gamma-1) \rho_{0}}{c_{0}^{4}}\left(c_{0}^{2} L_{p}+L_{\rho} \int d z\right)-\frac{1}{c_{0}^{2}} \quad 0 \quad 0\right) .
$$

Analogously, the projecting rows which subdivide velocity specifying the Alfvén modes are determined by the systems

$$
\begin{aligned}
& P_{1}\left(\begin{array}{l}
\rho^{\prime} \\
v_{x} \\
v_{y} \\
v_{z} \\
p^{\prime} \\
B_{x}^{\prime} \\
B_{y}^{\prime}
\end{array}\right)=v_{y, 1}, \\
& P_{2}\left(\begin{array}{l}
\rho^{\prime} \\
v_{x} \\
v_{y} \\
v_{z}^{\prime} \\
p^{\prime} \\
B_{x}^{\prime} \\
B_{y}^{\prime}
\end{array}\right)=v_{y, 2}
\end{aligned}
$$

and take the forms

$$
\begin{aligned}
& P_{1}=\left(\begin{array}{lllllll}
0 & 0 & \frac{1}{2} & 0 & 0 & 0 & -\frac{C_{A}}{2 B_{0}}
\end{array}\right), \\
& P_{2}=\left(\begin{array}{lllllll}
0 & 0 & \frac{1}{2} & 0 & 0 & 0 & \frac{C_{A}}{2 B_{0}}
\end{array}\right) .
\end{aligned}
$$

Projectors $P_{1}, P_{2}$ are also evaluated with accuracy up to terms proportional to the first powers of $L_{p}$ and $L_{\rho}$. When $P_{1}$, $P_{2}, P_{7}$ apply at the linearized equation (4), they reduce all therms containing perturbations which do not belong to the corresponding mode. Application of projectors yield density corresponding to the entropy mode is determined by the system of seven algebraic equations:

$$
P_{7}\left(\begin{array}{l}
\rho^{\prime} \\
v_{x} \\
v_{y} \\
v_{z} \\
p^{\prime} \\
B_{x}^{\prime} \\
B_{y}^{\prime}
\end{array}\right)=\rho_{7}
$$

It takes the form [19]

the linear dynamic equations which govern $v_{y, 1}, v_{y, 2}$, and $\rho_{7}$, respectively. In the following section, we consider the nonlinear excitation of these modes in the field of intense magnetoacoustic waves.

\section{Nonlinear Effects Which Associate with the Intense Magnetoacoustic Wave}

Among variety of nonlinear phenomena, we should distinguish nonlinear effects associated with the individual magnetoacoustic wave without accounting for its interaction with other modes and these ones which reflect interaction between different modes. In the context of nonlinear acoustics, distortions and nonlinear phenomena of intense wave are of the major importance. That means that magnetosonic perturbations are larger than that of other modes, at least in some temporal and special domains under consideration. We suppose that only one magnetosonic wave is dominant with the linear speed $C$ which is arbitrary of four roots of (10). The dynamic equation which describes the nonlinear distortion of magnetoacoustic wave and its nonlinear phenomena follow by means of application of corresponding projector at system (4). Since (4) include only quadratic nonlinear terms, the resulting dynamical equations are valid with accuracy up to quadratic nonlinear terms, also up to these one which stand by $L_{p}, L_{\rho}, L_{p p}, L_{p \rho}$, and $L_{\rho \rho}$.

The correct description of nonlinear effects associating with quadratic nonlinear terms imposes that the linear relations of perturbations in a wave should be corrected by means of involving terms which make them isentropic in the leading order [18]. The corrected links in terms of the longitudinal velocity $v_{z}$ are as follows:

$$
\psi_{3,4,5,6}^{c o r}=\left(\begin{array}{l}
\rho^{\prime} \\
v_{x} \\
v_{y} \\
v_{z} \\
p^{\prime} \\
B_{x}^{\prime} \\
B_{y}^{\prime}
\end{array}\right)_{3,4,5,6}
$$




$$
=\left(\begin{array}{c}
\frac{\rho_{0}}{C}-\frac{\rho_{0} S\left(c_{0}^{2} L_{p}+L_{\rho}\right)}{C^{2} c_{0}^{2}} \int d z \\
0 \\
\frac{c_{0}^{2} C_{A, z}}{C^{2} C_{A, x}}-\frac{C_{A, z}}{C_{A, x}}-\frac{C_{A, z}\left(c_{0}^{2} L_{p}+L_{\rho}\right)(\gamma-1+2 S)}{C_{A, x}^{3}} \int d z \\
\frac{c_{0}^{2} \rho_{0}}{C}-\frac{\left(c_{0}^{2} L_{p}+L_{\rho}\right)(\gamma-1+S)}{C^{2}} \int d z \\
\frac{\left(C^{2}-c_{0}^{2}\right) B_{0}}{C C_{A} C_{A, x}}+B_{0} \frac{\left(c_{0}^{2} L_{p}+L_{\rho}\right)\left(c_{0}^{2}(\gamma-1)+S\left(C^{2}+c_{0}^{2}\right)\right)}{C^{2} c_{0}^{2} C_{A} C_{A, x}} \int d z \\
0
\end{array}\right) v_{z, 3,4,5,6}+\left(\begin{array}{c}
A_{1} \\
A_{2} \\
0 \\
0 \\
A_{3} \\
A_{4} \\
0
\end{array}\right) v_{z, 3,4,5,6}^{2},
$$

where

$$
\begin{aligned}
& A_{1}=\frac{\left(c_{0}^{2}+C^{4}(\gamma-4)-C^{2} C_{A}^{2}(\gamma-3)\right) \rho_{0}}{4 C^{4}\left(c_{0}^{2}+C_{A}^{2}-2 C^{2}\right)}, \\
& A_{2}=\frac{c_{0}^{2}\left(C^{2}-c_{0}^{2}\right)\left(c_{0}^{4}-C^{2}\left(2 c_{0}^{2}+(\gamma-1) C_{A}^{2}\right)+\gamma C^{4}\right)}{2 C^{5}\left(C^{2}-C_{A}^{2}\right)\left(c_{0}^{2}+C_{A}^{2}-2 C^{2}\right)} \frac{C_{A, z}}{C_{A, x}}, \\
& A_{3}=\frac{c_{0}^{2}\left(c_{0}^{4}-3 \gamma C^{4}+C^{2}\left(2 c_{0}^{2}(\gamma-1)+C_{A}^{2}(\gamma+1)\right)\right) \rho_{0}}{4 C^{4}\left(c_{0}^{2}+C_{A}^{2}-2 C^{2}\right)}, \\
& A_{4}=\frac{B_{0}\left(C^{2}-c_{0}^{2}\right)\left(C^{6}-C^{2} c_{0}^{4}-C^{4}\left(3 C_{A, z}^{2}+c_{0}^{2}(\gamma-3)\right)+c_{0}^{2} C_{A, z}^{2}\left(C_{A, z}^{2}(\gamma+1)-c_{0}^{2}\right)\right)}{4 C^{2}\left(C^{4}-c_{0}^{2} C_{A, z}^{2}\right) C_{A} C_{A, x}^{3}} .
\end{aligned}
$$

Relations (25) may be obtained from the set of algebraic equation. Substituting into (4), they must yield the equivalent dynamic quadratic nonlinear equations for the MHD velocity $v_{z}(z, t)$. In fact, these four algebraic equations determine four unknowns $A_{1}, \ldots, A_{4}$. The weakly nonlinear equation which governs the component of velocity $v_{z}$ in all MHD wave modes takes the form

$$
\frac{\partial v_{z}}{\partial t}+C \frac{\partial v_{z}}{\partial z}+C D v_{z}+\varepsilon v_{z} \frac{\partial v_{z}}{\partial z}=0
$$

where

$$
\begin{aligned}
D & =\frac{S}{C c_{0}^{2}}\left(c_{0}^{2} L_{p}+L_{\rho}\right), \\
\varepsilon & =\frac{3 c_{0}^{2}+C_{A}^{2}(\gamma+1)-C^{2}(\gamma+4)}{2\left(c_{0}^{2}-2 C^{2}+C_{A}^{2}\right)} .
\end{aligned}
$$

It may be readily obtained by application of one from four magnetosonic projectors, $P_{3,4,5,6}$, and it was firstly derived by other method in [11, 12]. Equation (27) describes individual dynamics of fast and slow magnetosonic perturbations. It does not reflect the nonlinear interaction between different modes. In the absence of magnetic field, these amendments coincide with the well-known terms which make the progressive Riemann's wave isentropic [20]. This is the case $B_{0}=0$, $C=c_{0}, C_{A}=C_{A, x}=C_{A, z}=0$, and

$$
\begin{aligned}
& A_{1}=-\frac{(\gamma-3) \rho_{0}}{4 c_{0}^{2}}, \\
& A_{2}=0, \\
& A_{3}=\frac{\gamma+1}{4} \rho_{0}, \\
& A_{4}=0 .
\end{aligned}
$$

Equation (27) describes the nonlinear propagation of any MHD wave taken alone. It recalls dynamic equations for perturbations in other media which may be acoustically active [6, 7]. Equation (27) without accounting for heatingcooling function has been firstly derived and analyzed in the context of propagation of a saw-tooth impulse in [10]. Equation (27) may be readily rearranged into the leadingorder pure nonlinear equation, if $D \neq 0$ :

$$
\frac{\partial V}{\partial Z}-\frac{\varepsilon}{C^{2}} V \frac{\partial V}{\partial \tau}=0
$$


by means of new variables

$$
\begin{aligned}
V & =v_{z} \exp (D z), \\
Z & =\frac{1-e^{-D z}}{D}, \\
\tau & =t-\frac{z}{C} .
\end{aligned}
$$

Equation (30) may be solved by the method of characteristics. Note that $Z$ is always positive for nonzero $D$. It has been established that discontinuity in the waveform always forms in acoustically active media (that is the case of $D<0$ ) and may not arise otherwise due to attenuation [6].

As for the Alfvén modes, they are not excited by the magnetosonic modes in the leading order. This has been discovered by Nakariakov and coauthors in [11]. The reason is that the Alfvén modes are determined exclusively by $v_{y}$ and $B_{y}^{\prime}$, while the magnetoacoustic mode is determined by all other field perturbations. Magnetoacoustic and Alfvén modes only weakly couple in the nonlinear flows. That means that there are only mixed quadratic nonlinear terms which may form "the driving forces" in interactions between modes and hence have impact on the nonlinear dynamics. The projecting also confirms conclusions about weak coupling of magnetosonic and Alfvén modes.

Application of $P_{7}$ at the system (4) leads to weakly nonlinear evolutionary equation with properly distributed quadratic nonlinear terms. By means of projecting equation (1) into the different subspaces which are designated by dispersion relations, one readily obtains the equation describing dynamics of excess density which specifies the entropy mode, $\rho_{7}$. The row $P_{7}$ projects the system onto relative dynamic equation, eliminating all foreign terms in the linear part of the final equation. Among all variety of quadratic nonlinear terms, only MHD terms belonging to one from four wave modes are kept. They form the magnetoacoustic forces of magnetosonic heating or cooling. As a result of application of $P_{7}$, one arrives at the equation which governs an excess density in the entropy mode:

$$
\begin{aligned}
& \frac{\partial \rho_{7}}{\partial t}+\frac{(\gamma+1) L_{\rho}}{c_{0}^{2}} \rho_{7}=\frac{(\gamma-1)\left(C_{A, x} C^{4}+C_{A, z}\left(C^{2}-c_{0}^{2}\right)^{2}\right)\left(3 c_{0}^{2}+(\gamma+1) C_{A}^{2}-(\gamma+4) C^{2}\right)}{4 C_{A, x} C^{4} c_{0}^{2}\left(c_{0}^{2}+C_{A}^{2}-2 C^{2}\right)}\left(c_{0}^{2} L_{p}+L_{\rho}\right) \rho_{0} v_{z}^{2} \\
& \quad-\frac{\rho_{0}}{4 C^{2} c_{0}^{2}\left(C^{4}-c_{0}^{2} C_{A, z}^{2}\right)}\left(( \gamma - 1 ) \left(C^{2} c_{0}^{2}(4-\gamma)\left(c_{0}^{2} L_{p}+L_{\rho}\right)\right.\right. \\
& \quad+C^{4}\left(L_{\rho}+2 \rho_{0} c_{0}^{2} L_{p p}+2 \rho_{0} L_{\rho \rho}+c_{0}^{2}\left((2 \gamma-1) L_{p}+4 \rho_{0} L_{p \rho}\right)\right) \\
& \left.\quad-c_{0}^{2} C_{A, z}^{2}\left((5-\gamma) L_{\rho}+2 c_{0}^{4} \rho_{0} L_{p p}+2 \rho_{0} L_{\rho \rho}+c_{0}^{2}\left((\gamma+3) L_{p}+4 \rho_{0} L_{p \rho}\right)\right)\right) v_{z}^{2}+2\left(2 C^{4} \gamma(\gamma-1)-C^{2} c_{0}^{2}(\gamma-1)^{2}\right. \\
& \left.\left.\quad-C_{A, z}^{2} c_{0}^{2}\left(\gamma^{2}-1\right)\right)\left(c_{0}^{2} L_{p}+L_{\rho}\right) \frac{\partial v_{z}}{\partial z} \int v_{z}\left(z^{\prime}, t\right) d z^{\prime}\right) .
\end{aligned}
$$

Excitation of an excess density in the entropy mode is described by (32), where $C$ is one of four solutions to (27), that is, the speed of slow or fast MHD wave. Equation (32) includes only instantaneous perturbations and it is valid in description of heating excited by any type of magnetoacoustic force which is represented by its right-hand side. Equation (32) is written on in terms of excess density which specifies the entropy mode. The entropy mode is isobaric in accordance with (19). An excess temperature in the isobaric processes readily follows from the Clapeyron equation,

$$
\frac{\rho_{7}}{\rho_{0}}=-\frac{T_{7}}{T_{0}} .
$$

Isobaric cooling of a media is always followed by enlargement in its excess density, and isobaric heating corresponds to negative excess density.

3.1. Magnetoacoustic Heating for Different Cases of HeatingCooling Function. Equation (32) is much more simple for analysis in the case of periodic or quasiperiodic magnetoacoustic perturbations. In the leading order,

$$
\overline{v_{z}^{2}}=-\overline{\frac{\partial v_{z}}{\partial z} \int v_{z} d z}
$$

where top line denotes the temporal average over period of the magnetoacoustic wave. We reproduce averaged over the sound period form of (32) in some physically meaningful cases. The first example refers to the case when the heatingcooling function depends exclusively on temperature, $L=$ $L(T)$. In this particular case, one may readily rearrange (32) into the dynamic equation

$$
\begin{aligned}
& \frac{\overline{\partial \rho_{7}}}{\partial t}-\frac{(\gamma+1)}{C_{V}(\gamma-1) \gamma \rho_{0}} L_{T} \overline{\rho_{7}} \\
&= \frac{(\gamma-1)\left(3 C_{A}^{4}+2(\gamma+1) c_{0}^{4}+(3 \gamma+7) c_{0}^{2} C_{A}^{2}\right)}{4 \gamma c_{0}^{2}\left(c_{0}^{2}+C_{A}^{2}\right)^{2} C_{V}} \\
& \cdot L_{T} \overline{v_{z}^{2}},
\end{aligned}
$$


if $C_{A, z}=0$, that is, in the case of magnetic strength perpendicular to the wave vector, when speeds of fast modes equal $C= \pm \sqrt{c_{0}^{2}+C_{A}^{2}}$, and

$$
\frac{\overline{\partial \rho_{7}}}{\partial t}-\frac{(\gamma+1) L_{T}}{C_{V}(\gamma-1) \gamma \rho_{0}} \overline{\rho_{7}}=\frac{\gamma^{2}-1}{2 \gamma c_{0}^{2} C_{V}} L_{T} \overline{v_{z}^{2}},
$$

in the case of parallel propagation of sound (this corresponds to $C_{A, z}=C_{A}$ and fast modes with speeds $C= \pm c_{0}$ ). They are readily integrated for zero initial condition with the result

$$
\begin{aligned}
\overline{\rho_{7}}= & \exp \left(\frac{(\gamma+1) L_{T}}{C_{V}(\gamma-1) \gamma \rho_{0}} t\right) \\
& \cdot \int_{0}^{t} \exp \left(-\frac{(\gamma+1) L_{T}}{C_{V}(\gamma-1) \gamma \rho_{0}} t^{\prime}\right) I\left(z, t^{\prime}\right) d t^{\prime},
\end{aligned}
$$

where $I(z, t)$ is the averaged over magnetosonic period righthand force. This solution corresponds to zero initial $\rho_{7}$. In turn, $v_{z}(z, t)$ is a solution to (27). It may be approximately let sinusoidal function with constant $I$ proportional to $\overline{v_{z}^{2}}=$ $0.5 V_{0}^{2}$, where $V_{0}$ is the amplitude of $v_{z}$. In particular, (36) takes the form

$$
\overline{\rho_{7}}=\left(\exp \left(\frac{(\gamma+1) L_{T}}{C_{V}(\gamma-1) \gamma \rho_{0}} t\right)-1\right) \frac{V_{0}^{2}(\gamma-1)^{2} \rho_{0}}{4 c_{0}^{2}} .
$$

The case $L_{T}<0$ reflects the enlargement in the background temperature which achieves some maximum with time which is independent on $L_{T}$ :

$$
\overline{T_{7}}=\frac{V_{0}^{2}(\gamma-1)^{2} T_{0}}{4 c_{0}^{2}}
$$

If $L_{T}>0$, a gas is acoustically active. Linear conditions of isentropic and thermal instability in this particular case overlap. Sound enhances in a medium taking energy from the background. This is followed by isobaric cooling of a gas which is associated with enlargement of excess density specifying the entropy mode in time, $\rho_{7}>0$, and $T_{7}<$ 0 . Magnetoacoustic forces in (35), (36) are positive, and theoretically they make excess entropy density to increase with time infinitely. This growth in fact is suppressed by the nonlinear transfer of energy between different modes in dependence on the ratio of their intensities and nonlinear attenuation of the wave modes. In (35), (36), the second-order derivative $L_{T T}$ is omitted. That is valid if

$$
\left|L_{T T}\right| \ll \frac{(\gamma-1) \gamma C_{V}}{c_{0}^{2}}\left|L_{T}\right|=\frac{\gamma^{2}}{T_{0}}\left|L_{T}\right|
$$

The dynamic equation becomes simple in the case of constant heating per unit mass $\left(L_{p}=0\right)$. In the cold molecular gas, the external heating occurs mainly due to grain photoelectrons and cosmic rays [3]. This is the case of $L_{p}=0$. Dropping terms standing by the second derivatives $L_{p p}, L_{\rho \rho}$, and $L_{p \rho}$, we rearrange (27) as

$$
\begin{aligned}
\frac{\overline{\partial \rho_{7}}}{\partial t}+\frac{(\gamma+1) L_{\rho}}{c_{0}^{2}} \overline{\rho_{7}} \\
=\frac{(\gamma-1)\left(3 C_{A}^{4}+2(2 \gamma-1) c_{0}^{4}+(5 \gamma+3) c_{0}^{2} C_{A}^{2}\right) \rho_{0}}{4 c_{0}^{4}\left(c_{0}^{2}+C_{A}^{2}\right)^{2}} \\
\quad \cdot L_{\rho} \overline{v_{z}^{2}}
\end{aligned}
$$

in the case of magnetic strength perpendicular to the wave vector and the force induced by the fast modes and

$$
\frac{\overline{\partial \rho_{7}}}{\partial t}+\frac{(\gamma+1) L_{\rho}}{c_{0}^{2}} \overline{\rho_{7}}=\frac{(\gamma-1)(2 \gamma-1) \rho_{0}}{2 c_{0}^{4}} L_{\rho} \overline{v_{z}^{2}}
$$

in the case of magnetic strength parallel to the wave vector and the force generated by the fast modes. Equations (41) and (42) are readily integrated for zero initial condition with the result

$$
\begin{aligned}
\overline{\rho_{7}}= & \exp \left(-\frac{(\gamma+1) L_{\rho}}{c_{0}^{2}} t\right) \\
& \cdot \int_{0}^{t} \exp \left(\frac{(\gamma+1) L_{\rho}}{c_{0}^{2}} t^{\prime}\right) I\left(z, t^{\prime}\right) d t^{\prime},
\end{aligned}
$$

with $I$ standing in place of magnetoacoustic force of heating. The conditions of acoustic and thermal instabilities sound as $L_{\rho}>0$ and $L_{\rho}<0$.

The example of $L$ which depends exclusively on pressure is also of importance. In particular, it specifies heating by coronal current dissipation. In this case, $L_{\rho}=0$ and (32) may be rearranged in the dynamic equation

$$
\frac{\overline{\partial \rho_{7}}}{\partial t}=\frac{(\gamma-1)\left(3 C_{A}^{4}+2 \gamma c_{0}^{4}+(3 \gamma+5) c_{0}^{2} C_{A}^{2}\right) \rho_{0}}{4 c_{0}^{2}\left(c_{0}^{2}+C_{A}^{2}\right)^{2}} L_{p} \overline{v_{z}^{2}}
$$

in the case of perpendicular direction of magnetic strength and

$$
\frac{\overline{\partial \rho_{7}}}{\partial t}=\frac{\gamma(\gamma-1) \rho_{0}}{2 c_{0}^{2}} L_{p} \overline{v_{z}^{2}}
$$

in the case of parallel direction of magnetic strength. The magnetic gas is thermally neutral, but it is acoustically active if $L_{p}>0$. That ensures positive magnetoacoustic forces in the right-hand sides of (44) and (45) and enlargement in time of entropy excess density which follows an isobaric cooling of a gas. Equations (44) and (45) are readily integrated with respect to time.

\section{Concluding Remarks}

The main result of this study is analysis of the nonlinear instantaneous dynamic equation (32), which describes excitation of the excess density in the entropy mode in the 
field of intense magnetosonic waves. This is the nonlinear phenomenon which takes place in flows with irreversible internal losses or/and due to inflow of energy into a system. We consider nonadiabaticity due to external heating-cooling balance exclusively. For proper description of the magnetoacoustic heating (or cooling), the dynamic equation which governs perturbations in the entropy mode is extracted from the general system of equations by means of projecting. The projecting in fact eliminates perturbations of foreign modes in the linear part of equation but results to coupling terms which form "the driving force" responsible for the nonlinear excitation. The procedure is instantaneous and leads to the instantaneous dynamic equation (32). Its right-hand side represents the magnetoacoustic force of heating (cooling), manifesting two origins of the phenomenon, nonlinearity and nonadiabaticity. Equation (32) is valid for periodic and aperiodic sound. The only limitations are

(1) the weak nonlinearity of a flow, so that only quadratic nonlinear terms are considered;

(2) intense exciting wave perturbations as compared to that of other modes;

(3) weak attenuation (or enhancement) of wave mode over its characteristic period in the course of propagation.

The first condition means smallness of the Mach number, and the last condition ensures the wave process and specifies in fact the spectrum of magnetoacoustic disturbances. There is no restriction concerning strength of the magnetic field in this study.

Perturbation in density which specifies the entropy mode is followed by the isobaric perturbation in temperature which in turn forms the new background of the magnetoacoustic waves. As it always happens to the open systems, a gas may be acoustically active in dependence on the heatingcooling function. That means that a magnetic gas may be cooled by MHD wave perturbations. If the condition of acoustical activity is not valid, the temperature of a gas enlarges as it occurs in fluids due to irreversible losses, that is, shear, bulk viscosity, and thermal conductivity, though the reasons for damping are different and a flow is described by the different equations. The condition of thermal stability prevents unlimited theoretical growth of absolute excess temperature and density in the entropy mode. In fact, this enlargement is suppressed for other reasons. Not to mention attenuation, the dynamic equations should be rearranged in view of new ratio of magnitudes of specific perturbations. The flow is determined by a number of other factors, apart from the heating-cooling function. There are the angle between the magnetic strength and the wave vector, the equilibrium magnetic strength, and the equilibrium thermodynamic state of a gas. As for the magnetoacoustic waves in the accepted geometry of a flow, their individual nonlinear dynamics without accounting for interaction with other modes is analyzed analytically in detail in the number of papers; see [12] and the reference therein. The recent studies consider also impact of the second partial derivatives of $L$ on dynamics of MHD waves. The longitudinal velocity in MHD wave which contributes in the magnetosonic force must be solution to the corresponding dynamic equation (27).
Equations(35), (36), (41), (42), (44), and (45) are averaged over the period of magnetosonic perturbations for different kinds of $L$. They are valid for any angle between the equilibrium magnetic strength and wave number by means of linear magnetosonic speed $C$ which is one of roots of (10) and may correspond to slow or fast wave. For example, we have considered fast periodic magnetosonic perturbations and particular cases of the heating-cooling function:

(1) $L$ depends exclusively on temperature. In this case, the magnetoacoustic force of heating for perpendicular magnetic strength and wave vector is larger than in the case of parallel orientation, if $c_{0}>\sqrt{(2 \gamma-1) /(3-\gamma)} C_{A}$, that is, if the plasma $\beta$ is larger than $2(2 \gamma-1) /(\gamma(3-\gamma))\left(\beta=(2 / \gamma) c_{0}^{2} / C_{A}^{2}\right.$ is the ratio of hydrostatic and magnetic pressures). The ratio of magnetoacoustic forces tends to 1 when $c_{0} / C_{A}$ tends to infinity and achieves maximum $\left(4,5 \gamma^{3}+18 \gamma^{2}+30,5 \gamma+25\right) / 4(\gamma+1)(\gamma+$ $2)^{2}$. The next two cases refer to the heating-cooling function which depends on pressure and density, $L(p, \rho)$.

(2) $L_{p}=0 ; L_{\rho} \neq 0$. In the case, the ratio of "perpendicular" and "parallel" magnetoacoustic forces tends to 1 when $\beta$ tends to infinity achieving maximum value $\left(25 \gamma^{2}-18 \gamma+\right.$ $33) / 8(2 \gamma-1)(\gamma+2)$. The ratio is greater than unity if $\beta>$ $2(4 \gamma-5) / \gamma(7-3 \gamma)$.

(3) $L_{\rho}=0 ; L_{p} \neq 0$. The ratio of "perpendicular" and "parallel" magnetoacoustic forces also tends to 1 when $c_{0} / C_{A}$ tends to infinity and achieves maximum value $\left(9 \gamma^{2}+6 \gamma+\right.$ $25) / 8 \gamma(\gamma+2)$. It is greater than unity if $\beta>2(2 \gamma-3) / \gamma(5-\gamma)$. All estimations concern equal magnetoacoustic powers of "perpendicular" and "parallel" MHD perturbations (proportional to $\overline{v_{z}^{2}}$ ). All second derivatives of the heating-cooling function are dropped.

Magnetoacoustic heating in general leads to the nonuniformity of the equilibrium temperature of a plasma, that is, to the thermal lenses which in turn have impact on the speed of magnetosonic perturbations and dynamics of their wave fronts. These inhomogeneities may be of especial and substantive interest in the plasma's applications. The results of this study may be addressed to a hot atomic plasma with temperature greater than $10^{4} \mathrm{~K}$ and a cold molecular gas with temperature less than $10^{3} \mathrm{~K}$ and to different kinds of the function $L(p, \rho)$ which describes effects of nonadiabaticity. The radiation function also contributes in $L$. In particular, the optically thin hot plasma radiates proportionally to $\rho^{2-\eta} p^{\eta}$ [1]. Various models of coronal radiative losses and coronal heating may be found in [21] and references therein. The results may be useful in the inverse problems. The slow variations of equilibrium thermodynamic state may be measured remotely, which would provide data for analytical establishment of the kind of heating-cooling function in a plasma and magnetosonic perturbations. This may be of importance in many applications, also these relating to pulsed perturbations.

Theoretical predictions are of especial importance in view of the new studies which revealed existence of isentropically unstable regions in the solar corona with nonlinear enhancement of magnetoacoustic waves. The astrophysical media with adiabatic instability have been recently discussed 
in [22-28]. Papers $[24,26,28-33]$ were devoted to the features of the nonlinear magnetosound waves distortions and nonlinear interaction of waves in a magnetic fluid flow. The acoustical activity and nonlinear phenomena in acoustically active media have counterparts in many flows with other kind of anomalous thermodynamic relaxation corresponding dispersion: in flows of gases with excited vibrational degrees of molecules and chemically reacting gases. That is why the analytical methods and results may find application in the similar problems of fluid flows, both well-known as these less studied. The plasma applications are much more difficult in view of involving of magnetic equations and strong dependence of fluid dynamics on the angle between particles velocity and the magnetic strength. In particular, thermal self-action and self-refraction, which concern propagation of divergent beams with varying direction of velocity can not be considered by the analytical methods successful in the unmagnetized flows [34, 35].

\section{Data Availability}

No data were used to support this study.

\section{Conflicts of Interest}

The author declares that they have no conflicts of interest.

\section{References}

[1] J. F. Vesecky, S. K. Antiochos, and J. H. Underwood, "Numerical modeling of quasi-static coronal loops. I - Uniform energy input," The Astrophysical Journal, vol. 233, no. 3, pp. 987-997, 1979.

[2] R. B. Dahlburg and J. T. Mariska, "Influence of heating rate on the condensational instability," Solar Physics, vol. 117, no. 1, pp. 51-56, 1988.

[3] H. I. S. Miguel and A. Parravano, "On the thermal structure and stability of configurations with heat diffusion and a gainloss function. 3: Molecular gas," The Astrophysical Journal, vol. 424, no. 2, pp. 763-771, 1994.

[4] G. B. Field, “Thermal instability," The Astrophysical Journal, vol. 142, pp. 531-567, 1965.

[5] E. N. Parker, "Instability of thermal fields," The Astrophysical Journal, vol. 117, pp. 431-436, 1953.

[6] A. I. Osipov and A. V. Uvarov, "Kinetic and gasdynamic processes in nonequilibrium molecular physics," Soviet Physics Uspekhi, vol. 35, no. 11, pp. 903-923, 1992.

[7] N. E. Molevich, "Sound amplification in inhomogeneous flows of nonequilibrium gas," Acoustical Physics, vol. 47, no. 1, pp. 102105, 2001.

[8] N. S. Anderson, "Longitudinal magneto-hydrodynamic waves," The Journal of the Acoustical Society of America, vol. 25, no. 3, pp. 529-532, 1953.

[9] E. A. Ponomarev, "On the propagation of low-frequency oscillations along the magnetic field in a viscous compressible plasma," Soviet Astronomy, vol. 5, pp. 673-677, 1961.

[10] V. D. Sharma, L. P. Singh, and R. Ram, “The progressive wave approach analyzing the decay of a sawtooth profile in magnetogasdynamics," Physics of Fluids, vol. 30, no. 5, pp. 1572-1574, 1987.
[11] V. M. Nakariakov, C. A. Mendoza-Briceño, and M. H. Ibáñez S., "Magnetoacoustic waves of small amplitude in optically thin quasi-isentropic plasmas," The Astrophysical Journal, vol. 528, no. 2, pp. 767-775, 2000.

[12] R. Chin, E. Verwichte, G. Rowlands, and V. M. Nakariakov, "Self-organization of magnetoacoustic waves in a thermally unstable environment," Physics of Plasmas, vol. 17, no. 32, pp. 107-118, 2010.

[13] D. I. Zavershinsky, N. E. Molevich, and D. S. Ryashchikov, "Structure of acoustic perturbations in heat-releasing medium," Procedia Engineering, vol. 106, pp. 363-367, 2015.

[14] G. Brodin, L. Stenflo, and P. K. Shukla, "Nonlinear interactions between kinetic alfvén and ion-sound waves," Solar Physics, vol. 236, no. 2, pp. 285-291, 2006.

[15] D. I. Zavershinsky and N. E. Molevich, "Alfvén wave amplification as a result of nonlinear interaction with a magnetoacoustic wave in an acoustically active conducting medium," Technical Physics Letters, vol. 40, no. 8, pp. 701-703, 2014.

[16] O. Lyubchyk and Y. Voitenko, "Nonlocal nonlinear coupling of kinetic sound waves," Annales Geophysicae, vol. 32, no. 11, pp. 1407-1413, 2014.

[17] A. Perelomova, "On the nonlinear effects of magnetoacoustic perturbations in a perfectly conducting viscous and thermoconducting gas," Acta Physica Polonica A, vol. 130, no. 3, pp. 727733, 2016.

[18] A. Perelomova, "On the nonlinear distortions of sound and its coupling with other modes in a gaseous plasma with finite electric conductivity in a magnetic field," Archives of Acoustics, vol. 41, no. 4, pp. 691-699, 2016.

[19] A. Perelomova, "Magnetoacoustic heating in a quasi-isentropic magnetic gas," Physics of Plasmas, vol. 25, no. 4, p. 042116, 2018.

[20] O. V. Rudenko and S. I. Soluyan, Theoretical Foundations of Nonlinear Acoustics, Plenum, New York, NY, USA, 1977.

[21] R. Rosner, W. H. Tucker, and G. S. Vaiana, "Dynamics of the quiescent solar corona," The Astrophysical Journal, vol. 220, pp. 643-665, 1978.

[22] N. E. Molevich, D. I. Zavershinsky, R. N. Galimov, and V. G. Makaryan, "Traveling self-sustained structures in interstellar clouds with the isentropic instability," Astrophysics and Space Science, vol. 334, no. 1, pp. 35-44, 2011.

[23] R. Soler, J. L. Ballester, and S. Parenti, "Stability of thermal modes in cool prominence plasmas," Astronomy \& Astrophysics, vol. 540, A7, 2012.

[24] D. I. Zavershinskiy and N. E. Molevich, "Parametrical amplification of Alfvén waves in heat-releasing ionized media with magnetoacoustic instability," Astrophysics and Space Science, vol. 358, no. 1, p. 22, 2015.

[25] N. E. Molevich, D. S. Ryashchikov, and D. I. Zavershinskiy, "Influence of thermal conduction on MHD waves properties in thermally unstable plasma," Magnetohydrodynamics, vol. 52, no. 1, pp. 199-208, 2016.

[26] K. V. Krasnobaev, R. R. Tagirova, S. I. Arafailov, and G. Y. Kotova, "Evolution and saturation of Autowaves in photodissociation regions," Astronomy Letters, vol. 42, no. 7, pp. 460-473, 2016.

[27] D. S. Ryashchikov, N. E. Molevich, and D. I. Zavershinskii, "Characteristic times of acoustic and condensation instability in heat-releasing gas media," Procedia Engineering, vol. 176, pp. 416-422, 2017.

[28] K. V. Krasnobaev and R. R. Tagirova, "Isentropic thermal instability in atomic surface layers of photodissociation regions," 
Monthly Notices of the Royal Astronomical Society, vol. 469, no. 2, pp. 1403-1413, 2017.

[29] S. A. Belov, N. E. Molevich, and D. I. Zavershinskii, "Amplification of alfvén waves due to nonlinear interaction with a fast magnetoacoustic wave in acoustically active conductive media," Technical Physics Letters, vol. 44, no. 3, pp. 199-202, 2018.

[30] V. G. Makaryan and N. E. Molevich, "Stationary shock waves in nonequilibrium media," Plasma Sources Science and Technology, vol. 16, no. 1, pp. 124-131, 2007.

[31] N. E. Molevich, D. I. Zavershinskiy, and D. S. Ryashchikov, "Investigation of the MHD wave dynamics in thermally unstable plasma," Magnetohydrodynamics, vol. 52, no. 1, pp. 191-198, 2016.

[32] D. I. Zavershinsky and N. E. Molevich, "A magnetoacoustic autowave pulse in a heat-releasing ionized gaseous medium," Technical Physics Letters, vol. 39, no. 8, pp. 676-679, 2013.

[33] S. Kumar, V. M. Nakariakov, and Y.-J. Moon, "Effect of a radiation cooling and heating function on standing longitudinal oscillations in coronal loops," The Astrophysical Journal, vol. 824, no. 8, p. 8, 2016.

[34] A. Perelomova, "Thermal self-action effects of acoustic beam in a gas with reversible or irreversible chemical reaction," Acta Acustica united with Acustica, vol. 99, no. 3, pp. 352-358, 2013.

[35] A. Perelomova, "Self-refraction of acoustic pulses with shock fronts in some nonequilibrium media," Canadian Journal of Physics, vol. 92, no. 5, pp. 401-405, 2014. 


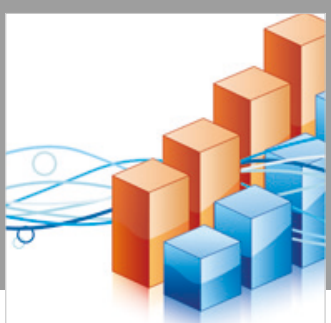

Advances in

Operations Research

\section{-n-m}
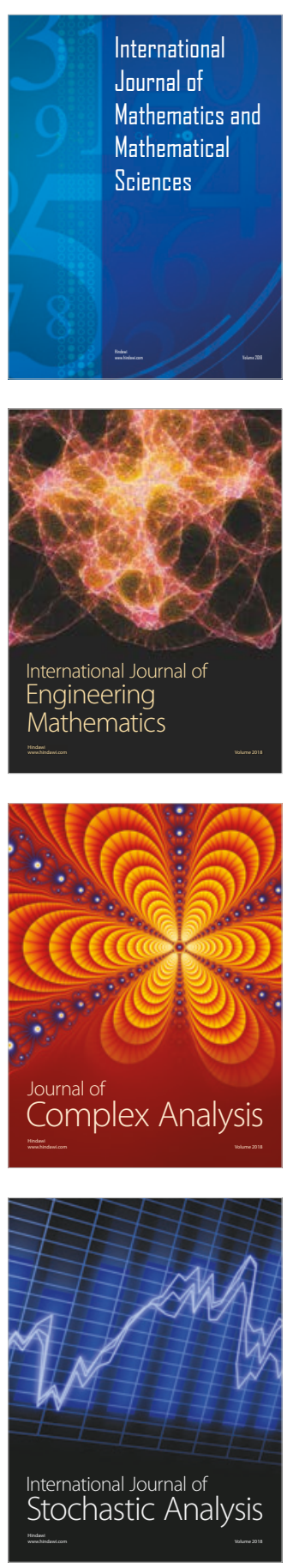
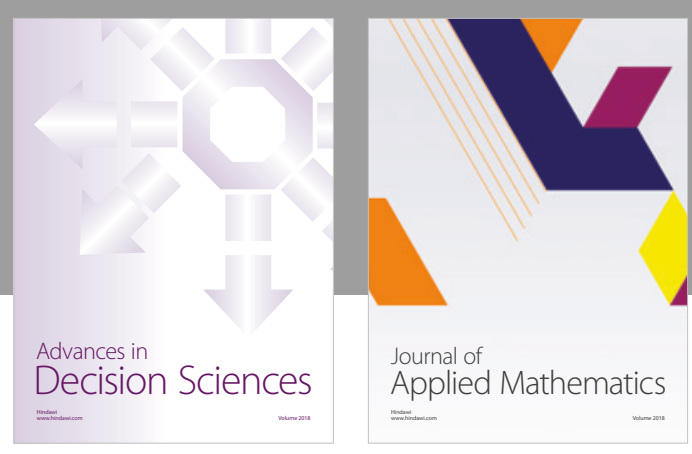

Journal of

Applied Mathematics
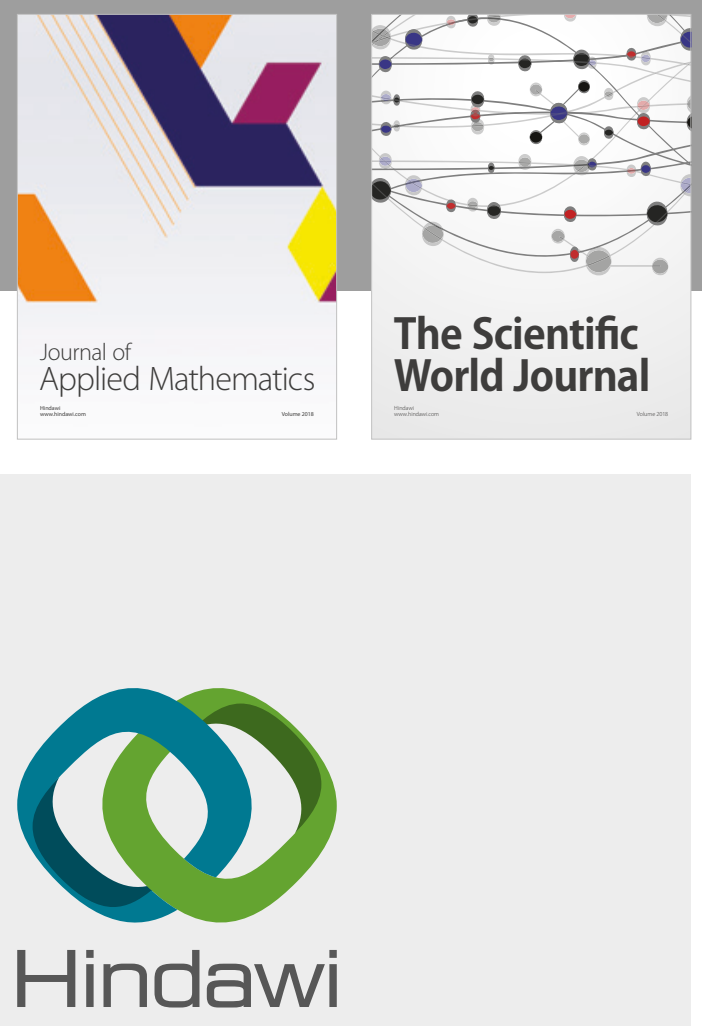

Submit your manuscripts at

www.hindawi.com

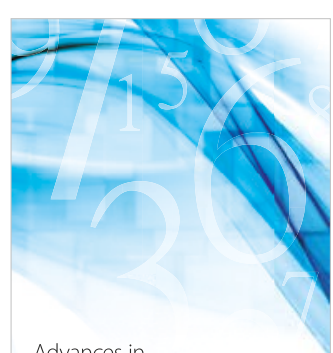

Advances in
Numerical Analysis
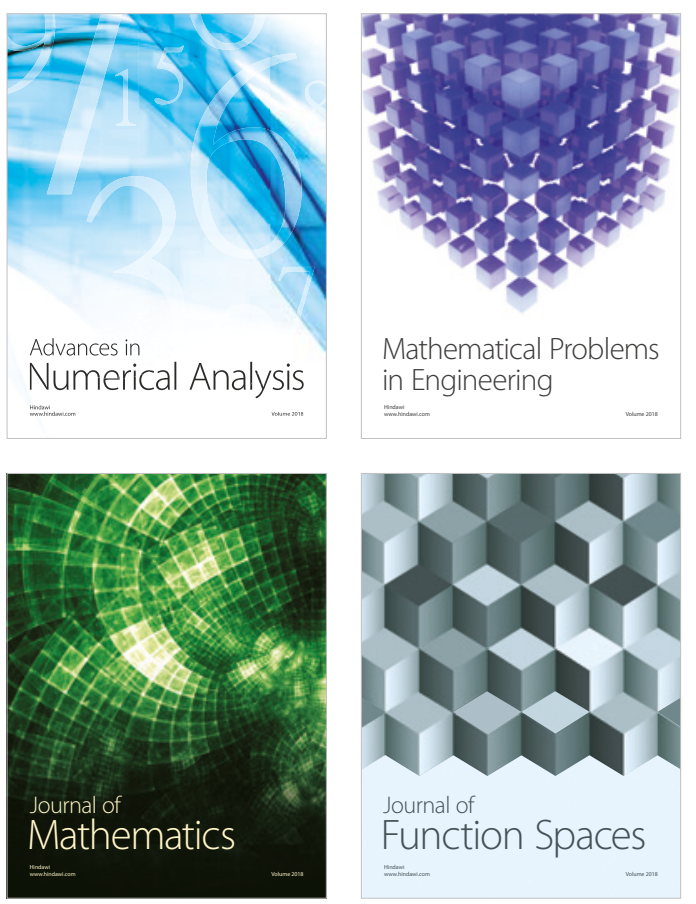

Mathematical Problems in Engineering

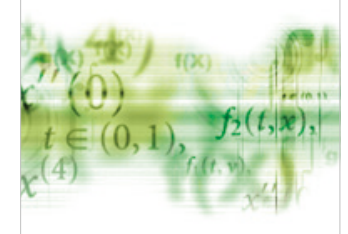

International Journal of

Differential Equations

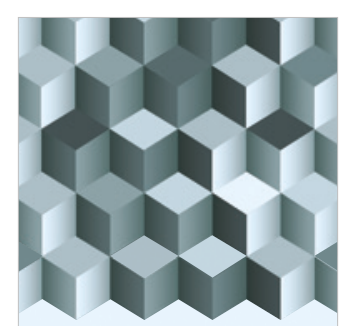

Journal of

Function Spaces
The Scientific

World Journal

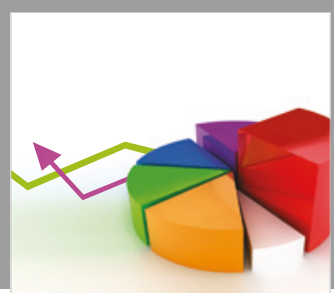

Journal of

Probability and Statistics
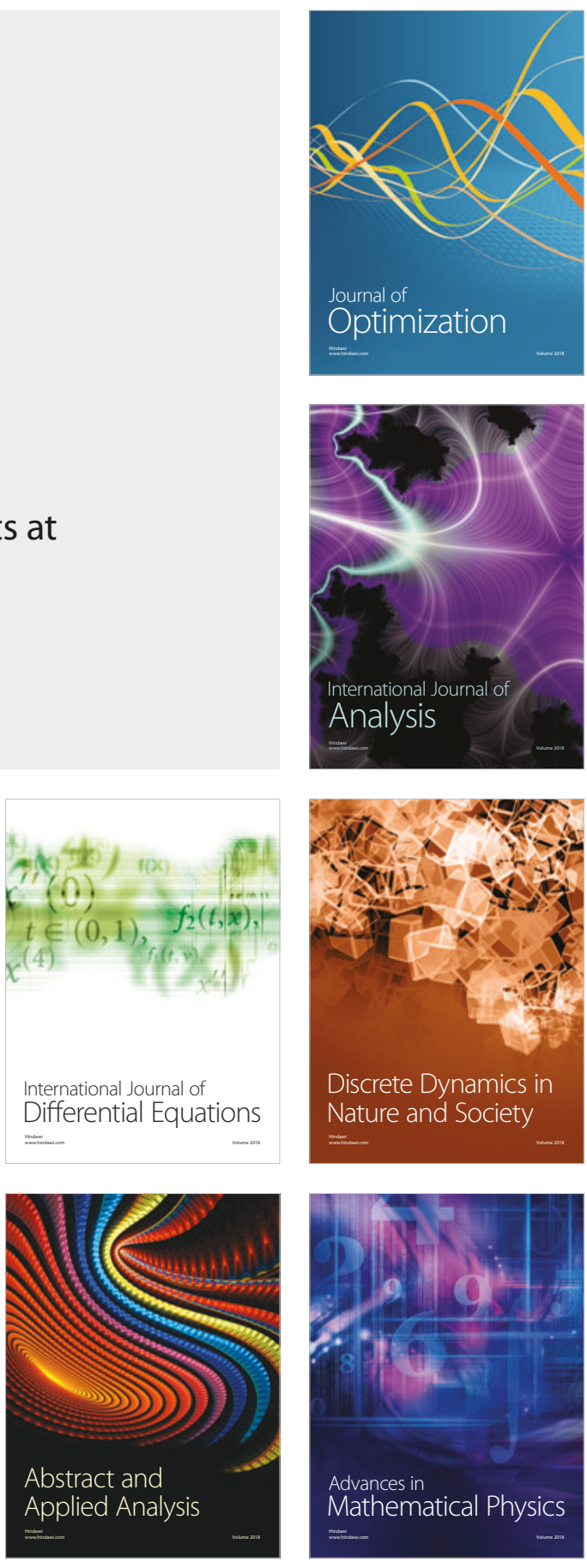